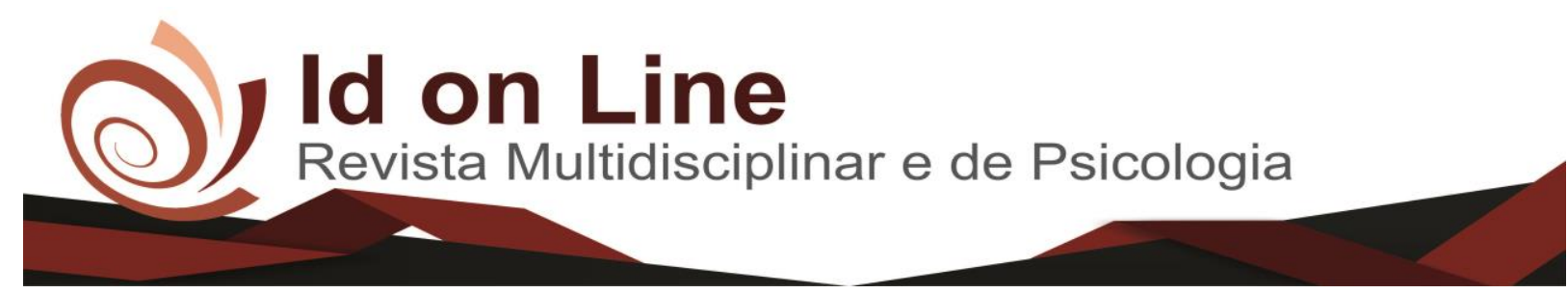

Artigo de Revisão

\title{
Lesões por Esforços Repetitivos e Distúrbios Osteomusculares em cirurgiões-dentistas
}

\author{
Hugo Vilela Viana ${ }^{1}$; Marcelo Pereira da Rocha ${ }^{2}$
}

\begin{abstract}
Resumo: As lesões por esforços repetitivos (LER) e distúrbios osteomusculares relacionados ao trabalho (DORT), em cirurgiões-dentistas, são de elevada prevalência e importante causa de afastamento do trabalho. Esta pesquisa, conduzida por meio de revisão da literatura, de forma sistemática, apresenta o perfil das publicações e os principais aspectos acerca das LER/DORT em dentistas. Os artigos selecionados foram acessados nas bases de dados Scielo, PubMed e Biblioteca Virtual de Saúde. Foi possível identificar as principais causas desses agravos, bem como medidas de prevenção, de modo que o profissional possa usufruir de melhor qualidade de vida. A organização do trabalho é importante fator de risco, capaz de causar ou agravar as condições musculoesqueléticas dos profissionais dos serviços odontológicos.
\end{abstract}

Palavras-chave: LER/DORT; Dentistas; Lesões por Esforço Repetitivo

\section{Repetitive Strain Injuries and Musculoskeletal Disorders in Dental Surgeons}

\begin{abstract}
Repetitive strain injuries (RSI) and work-related musculoskeletal disorders (DORS) in dental surgeons are of high prevalence and an important cause of withdrawal from work. This research, conducted through a review of the literature, systematically presents the profile of publications and the main aspects about RSI / DORT in dentists. The selected articles were accessed in the Scielo, PubMed and Virtual Health Library databases. It was possible to identify the main causes of these diseases, as well as prevention measures, so that the professional can enjoy a better quality of life. The organization of work is an important risk factor, capable of causing or aggravating the musculoskeletal conditions of dental professionals.
\end{abstract}

Keywords: LER / DORT; Dentists; Repetitive Strain Injury.

\section{Introdução}

As lesões por esforços repetitivos (LER) e distúrbios osteomusculares relacionadas ao trabalho (DORT) são agravos de elevada prevalência entre cirurgiões dentistas. Consistem em uma variedade de distúrbios músculoesqueléticos, geralmente

\footnotetext{
${ }^{1}$ Acadêmico do curso de Odontologia da Faculdade Independente do Nordeste; Contato: hugoviana_1@hotmail.com; Vitória da Conquista, Bahia, Brasil., Bairro boa vista n 47 ap 301 ed morada do parque contato (74)999452066

${ }^{2}$ Mestre em Saúde Coletiva, Especialista em Saúde Coletiva, em Planejamento e Práticas de Ensino e em Endodontia. Graduado em Odontologia e Biologia. Sanitarista efetivo da Secretaria Estadual de Saúde da Bahia (SESAB). Docente e Preceptor de cursos realizados pela SESAB. Membro Efetivo da Comissão Intergestora Regional. Docente do Curso de Odontologia da FAINOR.
} 
relacionados a tendões, a músculos ou articulações, bem como a algumas síndromes vasculares periféricas. Esses distúrbios geralmente afetam os membros superiores, costas e pescoço, embora os membros inferiores também possam estar envolvidos (HAYES; COCKRELL; SMITH, 2009).

Neste trabalho, foram utilizadas as expressões adotadas oficialmente pelos Ministérios da Saúde e da Previdência, do Brasil: lesões por esforços repetitivos (LER) e distúrbios osteomusculares relacionados ao trabalho (DORT), Elas designam um conjunto de agravos relacionados ao desgaste de estruturas do sistema musculoesquelético, comum em diversas categorias profissionais (MAENO et al.,2012).

A dor musculoesquelética é um problema de saúde do trabalhador para profissionais da odontologia, em decorrênciade longas jornadas, em posturas estáticas, usando movimentos precisos de mão e pulso por longos períodos (HAYE; COCKRELL; SMITH, 2009). Podem ocorrer a partir de um único evento, mas é mais frequente quando ocorre trauma acumulado, em função da utilização excessiva dos componentes do sistema músculoesquelético (PUTZ-ANDERSON; BERNARD; BURT, 1997).

Como as LER/DORT estão cada vez mais presentes entre profissionais da área de Odontologia, estes devem estar atentos aos primeiros sinais desses agravos, de modo a prevenir e tratá-las, o mais cedo possível, a fim de se evitar o comprometimento de estruturas anatômicas importantes, aumentando a longevidade laboral (PEREIRA; LOPES; OLIVEIRA, 2004).

As formas mais comuns de apresentação clínica dessas patologias em dentistas são as tendinites, tenossinovites, síndrome do túnel do carpo, miosites e bursites. Estas lesões podem causar dor, fadiga, queda de desempenho laboral, incapacidade temporária de exercer os movimentos (MARTINS; FADEL; RÉGIS-FILHO, 2003).

O surgimento dos sintomas, em geral, costuma levar a um imediato afastamento das atividades laborais e imobilização da região afetada. Repouso e acompanhamento médico são recomendados. Como os sintomas costumam surgir de forma lenta, é comum que os profissionais somente procurem algum auxilio quando o quadro já se mostra bastante grave, o que contribui para a evolução da doença (SANTOS, et al., 2007). 
As LER/DORT em cirurgiões-dentistas são bem descritas, bem como suas causas e formas de prevenção. No entanto, foi identificada a necessidade de estudos que busquem uma síntese do conhecimento na temática em questão, buscando-se identificar os principais aspectos das publicações. Este artigo tem por objetivo realizar uma revisão da literatura, acerca das LER/DORT em dentistas.

\section{Material e Métodos}

Este trabalho foi realizado por meio de revisão da literatura de forma sistemática, na qual se utilizam métodos definidos para identificar, selecionar e analisar criticamente os estudos (Ry's, 2009). A pesquisa foi conduzida a partir da seguinte indagação: quais são os principais aspectos abordados e resultados encontrados nos estudos acerca das LER/DORT em cirurgiões-dentistas? Para a busca dos artigos, foram utilizados inicialmente termos cadastrados nos Descritores em Ciências da Saúde: LER, DORT e dentista. Posteriormente, foram utilizados os termos em inglês: cumulative trauma disorders e dentist. O acesso foi realizado nas bases de dados Scielo, PubMed e Biblioteca Virtual de Saúde (BVS), espaço de congregação de fontes de informação em saúde na América Latina e Caribe, desenvolvido e administrado pela Bireme/Organização Panamericana de Saúde, em três idiomas, inglês, português e espanhol, no qual inclui bases de dados importantes, como Lilacs e Medline.

A inclusão dos trabalhos atendeu aos seguintes critérios: texto completo disponível, formato de artigo e atender a temática proposta. Não houve restrição de idioma de publicação. Foram excluídos trabalhos fora da temática proposta e publicados em formato distinto de artigo. Após a análise dos dados foi construído quadro com síntese dos artigos e apresentados os resultados, com o perfil das publicações e principais resultados dos estudos.

\section{Resultados}


Foram encontradas dez publicações, após busca realizada na BVS. Com o acesso realizado no Scielo não foi localizado nenhum trabalho. Por sua vez, com o acesso na PubMed foram encontrados seis trabalhos, totalizando 16 trabalhos disponíveis.

Após a leitura de títulos e resumos foram excluídos quatro trabalhos da BVS: um, devido à duplicidade, um devido ao fato de ser um editorial, outro, por ser uma carta dirigida a um leitor e um por não ter sido encontrado do texto completo. Das seis publicações da PubMed, foram excluídos três artigos, dois por terem sido encontrados anteriormente na BVS e outro, por fugir da temática proposta. Por fim, foram selecionados nove artigos para esta revisão, conforme quadro 1. 
QUADRo 1- SínTESE doS ARTIGOS SELECIONADOS PARA ESTE ESTUdo.

\begin{tabular}{|c|c|c|c|}
\hline Título & $\begin{array}{l}\text { Autores, periódico; local do estudo } \\
\text { e Base de dados }\end{array}$ & Metodologia & Principais resultados, conclusões/recomendações \\
\hline $\begin{array}{l}\text { Atividade ocupacional e prevalência de dor } \\
\text { osteomuscular em cirurgiões-dentistas de Belo } \\
\text { Horizonte, Minas Gerais, Brasil: contribuição ao } \\
\text { debate sobre os distúrbios osteomusculares } \\
\text { relacionados ao trabalho. }\end{array}$ & $\begin{array}{l}\text { Santos Filho, S B; Barreto, S M. } \\
\text { Cadernos de Saúde Pública; } 2001 . \\
\text { Belo Horizonte, Minas Gerais, Brasil. } \\
\text { Medline }\end{array}$ & $\begin{array}{l}\text { Foram utilizados questionários } \\
\text { em entrevistas com } 358 \\
\text { dentistas. Estudo de } \\
\text { prevalência de dor } \\
\text { osteomuscular e fatores } \\
\text { associados ao sintoma. }\end{array}$ & $\begin{array}{l}\text { Fatores associados à } \text { dor foram: pescoço: } \\
\text { ansiedade/depressão, ruído compressor, satisfação no } \\
\text { trabalho e uso de visão indireta; ombro: maior } \\
\text { produtividade, idade } 30-49 \text { anos; coluna: ansiedade } \\
\text { /depressão;). Há necessidade de estudos que aprofundem a } \\
\text { abordagem da queixa por região, incorporando entrevistas, } \\
\text { exames clínicos e complementares e realizando uma } \\
\text { exploração longitudinal da história ocupacional e de outras } \\
\text { exposições relevantes }\end{array}$ \\
\hline Prevalenceofmusculoskeletal disorders in dentists. & $\begin{array}{l}\text { Alexopoulos, E. C.; Stathi, I. C.; } \\
\text { Charizani, F. } \\
\text { BMC MusculoskeletDisorders. } 2004 . \\
\text { Salónica, Grécia. } \\
\text { Medline }\end{array}$ & $\begin{array}{l}\text { Pesquisa com questionários } \\
\text { entre } 430 \text { dentistas (resposta } \\
88 \% \text { em Salónica, na Grécia. } \\
\text { As questões incluem dados } \\
\text { sobre a carga de trabalho } \\
\text { física e psicossocial, a } \\
\text { necessidade de recuperação, a } \\
\text { percepção da saúde geral e a } \\
\text { ocorrência de queixas } \\
\text { musculoesqueléticas nos } \\
\text { últimos 12 meses, queixas } \\
\text { crônicas durante pelo menos } 1 \\
\text { mês, reclamações que levaram } \\
\text { a doença ausência, e a procura } \\
\text { de cuidados médicos. }\end{array}$ & $\begin{array}{l}62 \% \text { dos dentistas relataram pelo menos uma queixa } \\
\text { musculoesquelética, } 30 \% \text { das queixas foram crônicas, } 32 \% \\
\text { procuraram atendimento médico. Os fatores de carga física } \\
\text { relatados foram associados à ocorrência de dor na lombar, } \\
\text { dor no ombro e dor nas mãos / pulso. Com exceção das } \\
\text { queixas mão/pulso, os fatores físicos não foram associados } \\
\text { a queixas crônicas e ausência de doença } \\
\text { musculoesquelética. Não foi observada influência } \\
\text { consistente de fatores psicossociais em queixas, } \\
\text { cronicidade, ausência de doença e busca de cuidados } \\
\text { médicos. Uma percepção de saúde geral moderada foi um } \\
\text { fator significativo para queixas crônicas, comorbidade e } \\
\text { busca de cuidados médicos no qual o alto esforço percebido } \\
\text { foi significativo para o absenteísmo. Viver sozinho também } \\
\text { foi relacionado com o aumento do absenteísmo, devido a } \\
\text { dor no ombro e mão/pulso. A carga física é fator de risco } \\
\text { para a ocorrência de distúrbios musculoesqueléticos. Mais } \\
\text { de uma e queixas graves estão relacionadas à saúde geral } \\
\text { percebida. Os sintomas crônicos parecem determinar a } \\
\text { procura de cuidados médicos. As intervenções ergonômicas } \\
\text { podem ter um impacto maior na prevenção de reclamações } \\
\text { mão/pulso. }\end{array}$ \\
\hline
\end{tabular}




\begin{tabular}{|c|c|c|c|}
\hline $\begin{array}{l}\text { Lesões por esforços repetitivos/distúrbios } \\
\text { osteomusculares relacionados ao trabalho em } \\
\text { cirurgiões-dentistas }\end{array}$ & $\begin{array}{l}\text { Regis Filho, GI; Michels, G; Sell, I. } \\
\text { Revista Brasileira de Epidemiologia; } \\
2006 . \\
\text { Santa Catarina, Brasil. } \\
\text { Lilacs }\end{array}$ & $\begin{array}{l}\text { Estudo epidemiológico } \\
\text { transversal, com aplicação de } \\
\text { questionário. Relacionaram-se } \\
\text { as tarefas executadas pelo } \\
\text { cirurgião-dentista e as } \\
\text { LER/DORT. }\end{array}$ & $\begin{array}{l}\text { O sexo feminino apresenta mais patologias que o } \\
\text { masculino. Ombro/braço }(39,40 \%) \text {, punho/mão }(18,30 \%) \text { e } \\
\text { pescoço }(17,20 \%) \text {, são as regiões mais afetadas por } \\
\text { LER/DORT. As tarefas executadas expõem o profissional a } \\
\text { um risco considerável de adquirir LER/DORT. }\end{array}$ \\
\hline General health of dentists. Literature review. & $\begin{array}{l}\text { Puriene A.; Janulyte V.; Musteikyte } \\
\text { M.; Bendinskaite R. } \\
\text { General health of dentists. Literature } \\
\text { review. } \\
\text { Stomatologija. } 2007 . \\
\text { Medline }\end{array}$ & $\begin{array}{l}\text { Revisão da literatura acerca de } \\
\text { agravos relacionados ao } \\
\text { trabalho de ordem física e } \\
\text { psicológica, em dentistas. }\end{array}$ & $\begin{array}{l}\text { A vulnerabilidade dos dentistas a problemas de saúde } \\
\text { relacionada à prática odontológica é elevada. Em diferentes } \\
\text { países, os dentistas relataram ter uma saúde geral deficiente } \\
\text { e sofrem de vários problemas relacionados à saúde. O } \\
\text { dentista é sujeito a uma grande variedade de doenças físicas } \\
\text { e psicológicas induzidas ou agravada pelo ambiente de } \\
\text { trabalho. Eles afetam muito a saúde dos profissionais } \\
\text { dentários e a qualidade de vida do profissional. }\end{array}$ \\
\hline $\begin{array}{l}\text { Distúrbios osteomusculares e fatores associados em } \\
\text { cirurgiões dentistas do meio oeste do estado de Santa } \\
\text { Catarina }\end{array}$ & $\begin{array}{l}\text { Kotliarenko, A.; Michel-Crosato, E.; } \\
\text { Biazevic, M. G. H.; Crosato, E.; } \\
\text { Silva, P. R } \\
\text { Revista Odonto ciência. 2009. } \\
\text { Região Oeste de Santa Catarina, } \\
\text { Brasil. } \\
\text { Lilacs }\end{array}$ & $\begin{array}{l}\text { Estudo transversal, com } \\
\text { aplicação de questionário, com } \\
153 \text { cirurgiões-dentistas. }\end{array}$ & $\begin{array}{l}142(93 \%) \text { relataram ter tido distúrbios osteomusculares em } \\
\text { pelo menos uma parte do corpo no último ano, em } \\
\text { decorrência da atividade profissional. As regiões mais } \\
\text { prevalentes relatadas pelos profissionais foram coluna } \\
\text { cervical e dor nos ombros, acometendo } 107(70 \%) \text { e } 99 \\
(65 \%) \text { dos profissionais, respectivamente. A prevalência } \\
\text { dos distúrbios osteomusculares foi alta nesta amostra e } \\
\text { houve associação com algumas características ocupacionais } \\
\text { e socioeconômicas. }\end{array}$ \\
\hline $\begin{array}{l}\text { Estudo da prevalência de doenças ocupacionais em } \\
\text { Cirurgiões-Dentistas de São José dos Campos. }\end{array}$ & $\begin{array}{l}\text { Pereira, A. S.; Fonseca, M. F.; } \\
\text { Aizawa, L. H.; Ribeiro, C. F.; Torres, } \\
\text { C. R. G.; Pucci, C. R. } \\
\text { Odonto (São Bernardo do Campo); } \\
2011 . \\
\text { São José dos Campos, São Paulo, } \\
\text { Brasil } \\
\text { Lilacs }\end{array}$ & $\begin{array}{l}150 \text { dentistas responderam } \\
\text { questionários contendo } \\
\text { perguntas de caráter } \\
\text { psicológico, psicossocial, } \\
\text { história da saúde geral e } \\
\text { aspectos ergonômicos do } \\
\text { trabalho. }\end{array}$ & $\begin{array}{l}\text { A ocorrência de LER/DORT foi maior entre os } \\
\text { profissionais que apresentam uma qualidade de vida } \\
\text { insatisfatória, como horas de sono insuficiente, ausência de } \\
\text { atividades físicas, sobrecarga de trabalho, associada a um } \\
\text { número pequeno de intervalos. Ressalta-se a importância de } \\
\text { adotar um estilo de vida saudável associado à correta } \\
\text { organização ergonômica do trabalho, indispensáveis para a } \\
\text { qualidade e produção durante o exercício da profissão. }\end{array}$ \\
\hline
\end{tabular}




\begin{tabular}{|c|c|c|c|}
\hline Ergonomic applications to dental practice & $\begin{array}{l}\text { Gupta, S. } \\
\text { Indian Journal of } \\
\text { DentistriResearsh. } 2012 . \\
\text { Medline }\end{array}$ & $\begin{array}{l}\text { Revisão da literatura } \\
\text { acerca dos fatores de risco } \\
\text { associados a DORT e } \\
\text { ergonomia aplicada ao } \\
\text { trabalho de cirurgiões- } \\
\text { dentistas. }\end{array}$ & $\begin{array}{l}\text { Fatores de risco para DORT: estresse, má } \\
\text { flexibilidade, posicionamento inadequado, intervalos } \\
\text { infrequentes, movimentos repetitivos, músculos } \\
\text { posturais fracos, posturas difíceis, prolongadas e } \\
\text { ajuste inadequado do equipamento. O design } \\
\text { ergonômico apropriado é necessário para prevenir } \\
\text { lesões por esforços repetitivos, que podem se } \\
\text { desenvolver ao longo do tempo e levar a uma } \\
\text { incapacidade de longo prazo. Foram apresentadas } \\
\text { estratégias para prevenir LER/DORT. }\end{array}$ \\
\hline $\begin{array}{l}\text { Sintomas osteomusculares em cirurgiões- } \\
\text { dentistas da rede pública. }\end{array}$ & $\begin{array}{l}\text { Silva, H.P.L.; Jesus, C.S. } \\
\text { Revista AMIRGS; } 2013 . \\
\text { Jequié, Bahia, Brasil } \\
\text { Lilacs }\end{array}$ & $\begin{array}{l}\text { Estudo transversal, } \\
\text { descritivo, aplicando-se } \\
\text { questionário, com } 20 \\
\text { dentistas. }\end{array}$ & $\begin{array}{l}\text { Tempo médio de atuação, } 13,9 \text { anos; jornada média } \\
\text { de trabalho, } 41 \text { horas semanais; atendimento de mais } \\
\text { de } 14 \text { pacientes/dia }(75 \%) \text {. Dor musculoesquelética } \\
\text { com maior frequência na coluna cervical ( } 90 \%) \text { e } \\
\text { região lombar ( } 80 \%) \text {. Elevada prevalência de dor } \\
\text { osteomuscular, principalmente em regiôes cervical, } \\
\text { ombro e coluna. A organização do trabalho contribui } \\
\text { para exposição a fatores de riscos ergonômicos e o } \\
\text { desenvolvimento de DORT. }\end{array}$ \\
\hline $\begin{array}{l}\text { Musculoskeletal disorders and perception of } \\
\text { workingconditions: A survey of } \\
\text { Brazilian dentists in São Paulo }\end{array}$ & $\begin{array}{l}\text { Garbin, A. J. Í.; Soares, G. B.; } \\
\text { Arcieri, R. M.; Garbin, C. A. S.; } \\
\text { Siqueira, C. E } \\
\text { International Journal of } \\
\text { Occupanc i Medicine and } \\
\text { Environmental Health. } 2017 . \\
\text { Noroeste de São Paulo, Brasil } \\
\text { Medline }\end{array}$ & $\begin{array}{l}\text { Estudo transversal com } \\
204 \text { dentistas de clínicas de } \\
\text { saúde pública do noroeste } \\
\text { de São Paulo, Brasil. Os } \\
\text { dados foram coletados } \\
\text { através de entrevistas, } \\
\text { usando Questionário } \\
\text { Nórdico }\end{array}$ & $\begin{array}{l}(81,4 \%) \text { apresentaram distúrbios } \\
\text { musculoesqueléticos, especialmente no pescoço, } \\
\text { ombros e parte inferior das costas. Sintomas no } \\
\text { pescoço }(15,7 \%) \text {, ombros }(12,7 \%) \text { e parte inferior da } \\
\text { região lombar }(15,7 \%) \text { foram as principais causas de } \\
\text { absenteísmo nos últimos } 12 \text { meses. Os fatores de } \\
\text { risco ocupacional percebidos como os mais } \\
\text { problemáticos foram: dobrar a coluna de uma } \\
\text { maneira inadequada, continuar trabalhando na mesma } \\
\text { posiçãa por longos períodos. A comparação entre } \\
\text { dentistas sintomáticos e assintomáticos mostrou } \\
\text { diferença estatisticamente significante na percepção } \\
\text { de fatores de risco ocupacionais. Houve forte } \\
\text { correlação } \mathrm{t}(\mathrm{r}=0,697) \text { entre a intensidade da dor e o } \\
\text { escore total de incapacidade causada pela dor. }\end{array}$ \\
\hline
\end{tabular}

Fonte: Autor.

Id on Line Rev. Mult. Psic. V.11 N. 38. 2017 - ISSN 1981-1179

Edição eletrônica em http://idonline.emnuvens.com.br/id 


\section{Perfil dos trabalhos selecionados}

Os estudos analisados foram publicados (quadro 1) em distintos periódicos, não sendo encontrada mais de uma publicação em um mesmo veículo. Quanto à nacionalidade, seis $(85,7 \%)$ foram do Brasil e três estrangeiros. Predominaram as autorias múltiplas $(88,8 \%)$ e os textos em português $(55,5 \%)$. Quanto ao local de realização dos estudos nacionais, por região, três foram quatro foram provenientes do Sudeste, dois do Sul e um do Nordeste.

As bases de dados cujos artigos foram indexados são LILACS (4; 44,45\%); MEDLINE (5; 55,5\%). O período de publicação variou de 2001, a mais antiga a 2017, a mais recente.

Quanto à metodologia utilizada, dois estudos $(22,2 \%)$ foram baseados em revisões da literatura. Os demais $(7 ; 77,7 \%)$ foram baseados em entrevistas, com aplicação de questionário, nos quais os profissionais pesquisados responderam a aspectos sociais, demográficos, econômicos, sinais e sintomas de LER/DORT, dentre outros.

\section{Principais aspectos dos trabalhos}

Estudo realizado em Belo Horizonte, Brasil, apresentou inquérito epidemiológico, conduzido por meio de questionário, referente à queixa de dor osteomuscular no segmento superior em cirurgiões-dentistas. Fatores associados à dor foram: pescoço: ansiedade/depressão, ruído compressor, satisfação no trabalho e uso de visão indireta; ombro: maior produtividade, idade 30-49 anos; coluna: ansiedade/depressão. Os autores indicaram a necessidade de estudos futuros que aprofundem a abordagem da queixa por região, incorporando entrevistas, exames clínicos e complementares e realizando uma exploração longitudinal da história ocupacional e de outras exposições relevantes (SANTOS FILHO et al., 2001).

$\mathrm{Na}$ Grécia, em estudo realizado por meio de questionários entre 430 dentistas, foram investigadas as relações entre características físicas, psicossociais e individuais e queixas musculoesqueléticas de dor nas regiões lombar, pescoço, ombros, mão e pulso. $62 \%$ dos dentistas relataram pelo menos uma queixa musculoesquelética, sendo que 30\% das queixas foram crônicas. Dos que relataram alguma queixa, $32 \%$ procuraram atendimento médico. Os fatores de carga física relatados foram associados à ocorrência de dor nas costas, dor no 
ombro e dor nas mãos/pulso. Os fatores físicos não foram associados a queixas crônicas. Não foi observada influência consistente de fatores psicossociais em queixas, cronicidade, ausência de doença e busca de cuidados médicos. A carga física entre dentistas colabora na ocorrência de distúrbios musculoesqueléticos. As intervenções ergonômicas podem ter um impacto maior na prevenção de reclamações mão/pulso. Ao investigar a influência dos fatores de risco relacionados ao trabalho na saúde musculoesquelética, características psicossociais e outras características pessoais devem ser levados em consideração. (ALEXOPOULOS et al., 2004).

A relação entre as tarefas executadas pelo cirurgião-dentista e as LER/DORT foi avaliada em estudo conduzido por Regis Filho et al., (2006). Houve presença de associação estatisticamente significante entre os dois sexos e as patologias. $\mathrm{O}$ sexo feminino apresentou mais lesões que o masculino. Ombro/braço (39,40\%), punho/mão $(18,30 \%)$ e pescoço $(17,20 \%)$ foram as regiões mais afetadas. As LER/DORT chamam a atenção pelo aumento da sua incidência e por existirem evidências de sua associação com o ritmo de trabalho. $\mathrm{O}$ exercício profissional obriga que cirurgiões-dentistas utilizem na execução das tarefas os membros superiores e estruturas adjacentes, com repetitividade de um mesmo padrão de movimento, compressão mecânica das estruturas localizadas na região, assumindo posturas incorretas, utilizando força excessiva e trabalhando sob pressão temporal. As tarefas executadas expõem esses profissionais a um risco considerável de adquirir LER/DORT.

Puriene et al., (2007) realizaram revisão da literatura acerca dos agravos à saúde dos dentistas, decorrentes de suas atividades odontológicas. Ressaltaram grande variedade de doenças físicas e psicológicas induzidas ou agravada pelo ambiente de trabalho do dentista. A prevalência de queixas musculoesqueléticas entre dentistas, como entre outros profissionais de saúde, é alta e bem documentada. Também é elevada a frequência de absenteísmo, em decorrência das lesões musculoesqueléticas.

Com o intuito de registrar a prevalência dos distúrbios osteomusculares em cirurgiõesdentistas e estabelecer possíveis relações com fatores sociodemográficos e ocupacionais, foi realizado estudo transversal, com uso de instrumento de pesquisa autoaplicável para uma amostra de 153 profissionais de Santa Catarina, Brasil. 93\% dos dentistas relataram distúrbios osteomusculares em pelo menos uma parte do corpo, no último ano, em decorrência da atividade profissional. As regiões mais prevalentes foram coluna cervical e dor nos ombros, acometendo $107(70 \%)$ e $99(65 \%)$ dos profissionais, respectivamente. A prevalência dos 
distúrbios osteomusculares foi alta na amostra e houve associação com algumas características ocupacionais e socioeconômicas (KOTLIARENKO et al., 2009).

A prevalência de doenças ocupacionais entre dentistas foi avaliada por Pereira et al., (2011), na cidade de São José dos Campos, SP. Os pesquisadores distribuíram 150 questionários entre profissionais dentistas que atuavam tanto na rede pública quanto na privada, no município. De acordo com os dados levantados, $41 \%$ dos profissionais relataram sentir algum tipo de dor osteomuscular. Destes, 46,15\% tiveram o diagnóstico de LER. A maior parte dos casos de dor foi relatada na região envolvendo ombros e pescoço. Os profissionais com hábitos como tabagismo, ou que apresentavam estresse, foram os que mais relataram alguma dor.

Gupta (2012) discutiu estratégias ergonômicas que podem atuar na prevenção das dores osteomusculares em dentistas. De acordo com o autor, a posição da cadeira do dentista e a do paciente, o layout da estação de trabalho e o uso de apoio para os braços influenciam no conforto do profissional, e podem prevenir o aparecimento das dores. Ressaltou o design ergonômico apropriado como um fator necessário para prevenir lesões por esforços repetitivos, que podem se desenvolver ao longo do tempo e levar a uma incapacidade de longo prazo. Foram ainda apresentadas estratégias para prevenir LER/DORT.

No trabalho realizado por Silva e Jesus (2013) foram avaliados os sintomas osteomusculares apresentados por dentistas que trabalham na rede pública no município de Jequié, BA. Foram avaliados 20 profissionais, que responderam ao Questionário Nórdico de Sintomas Osteomusculares e um questionário com dados sociodemográficos e ocupacionais. De acordo com os dados obtidos, $90 \%$ dos profissionais apresentavam dor na coluna cervical, e $80 \%$ na região lombar. Estes dados apontam para elevada prevalência de dor osteomuscular entre estes profissionais, alertando para o risco de desenvolvimento de LER/DORT.

Um estudo transversal foi realizado com 204 dentistas, trabalhadores de clínicas de saúde pública do Noroeste de São Paulo, Brasil. Os dados foram coletados por meio de entrevistas, usando Questionário Nórdico. Os profissionais apresentaram alta prevalência de distúrbios musculoesqueléticos nas regiões de pescoço, ombros, coluna e punhos/mãos. Os dentistas com relato de dor identificaram melhor a presença de fatores de risco que aqueles que não relataram dor. Sintomas musculoesqueléticos presentes, tornam esses profissionais mais atentos à problemas relacionados à prática de sua profissão. As LER/DORT são causas importantes de absenteísmo entre profissionais da odontologia (ISPER et al., 2017).

\section{Discussão}


As patologias relacionadas ao trabalho como LER/DORT são caracterizadas pelo esforço repetitivo, e atingem grupos musculares durante a execução das atividades laborais. São caracterizadas por fortes dores, pela utilização de certos grupos musculares durante longos períodos. As dores muitas vezes podem ser incapacitantes, e acarretar o afastamento do profissional de suas atividades (SILVA, et al., 2012)

A repetição constante de movimentos e a postura inadequada durante o trabalho, principalmente dos membros superiores, com movimentos constantes de flexão e extensão do punho, além da compressão mecânica das bases da mão durante a utilização dos instrumentos, que muitas vezes apresentam estruturas inadequadas, constituem as principais causas para a presença de doenças ocupacionais em dentistas (SANTOS, et at., 2013)

Os resultados observados durante a execução deste trabalho demonstram a alta prevalência de DORT entre cirurgiões-dentistas. Em geral, foi possível observar que a dor se manifestou principalmente na região do membro superior, atingindo ombros e o braço utilizado durante a execução do trabalho. Muitos profissionais relataram fazer algum tipo de tratamento para a dor.

A postura incorreta e equipamentos odontológicos com layout inadequado são fatores que contribuem para o surgimento das dores em cirurgiões-dentistas (Régis-Filho et al.,) (2009). O comprometimento de grupos musculares durante a execução da atividade laboral é fator de risco ao desenvolvimento de LER/DORT (REGIS-FILHO; MICHELS; SELL, 2009).

Outro fator que deve ser levado em consideração é a presença de um ou mais auxiliares, principalmente durante a realização de cirurgias e outros procedimentos. A má postura ao tentar alcançar equipamentos e instrumentos, acúmulo de funções e excesso de esforço na realização de procedimentos poderiam ser atenuados com a presença de um profissional auxiliar no consultório. Um dos maiores motivos que levam ao surgimento do estresse é o excesso de trabalho, tanto entre profissionais que atuam na rede pública quanto entre os que trabalham na rede particular. Horas excessivas de trabalho, sem o adequado repouso acarretam estresse e perda de qualidade de vida.

Diversos aspectos referentes à profissão tornam os dentistas mais susceptíveis ao desenvolvimento de LER/DORT, comparando-se com outras profissões da área de saúde. O excesso de horas de trabalho, má postura e uso de equipamentos inadequados são fatores associados ao surgimento do problema. Aspectos relacionados à qualidade de vida, como estresse, tabagismo e sedentarismo também mostraram relação com esses agravos. 
Os estudos encontrados demonstram a gravidade do problema e a necessidade de prevenção desses agravos. Assim, é preciso ressaltar a importância da prevenção de lesões osteomusculares. Uso de equipamentos ergonomicamente adequados, intervalos durante o trabalho e prática de ginástica laboral são algumas das atitudes que podem auxiliar nesta prevenção.

.As pesquisas ainda carecem de aprofundamento quanto queixa por região, para melhor diagnóstico. Faz-se necessário conhecer a história ocupacional e outras exposições relevantes, de modo a se compreender melhor a relação entre o trabalho e o risco de adoecimento.

\section{Considerações Finais}

Os estudos demonstraram elevada prevalência de LER/DORT em dentistas, o que leva ao seu afastamento de atividades odontológicas. A organização do trabalho é importante fator de risco, capaz de provocar ou agravar as condições musculoesqueléticas. Foi possível identificar as principais causas desses agravos, bem como medidas de prevenção, de modo que o profissional possa usufruir de melhor qualidade de vida.

Com este trabalho foi possível conhecer o perfil das publicações indexadas em importantes bases de dados pesquisadas e compreender os seus principais aspectos, apesar das limitações do estudo, devido ao número reduzido de trabalhos encontrados, fato que aponta para a necessidade de mais pesquisas nessa temática.

\section{Referências}

ALEXOPOULOS, E. C.; STATHI, I. C.; CHARIZANI, F. Prevalence ofmusculoskeletaldisorders in dentists. BMC musculoskeletaldisorders, v. 5, n. 1, p. 16, 2004

GARBIN, A. J. Í. et al. Musculoskeletal disorders and perception of working conditions: a survey of brazilian dentists in São Paulo. International Journal of Occupational Medicine and Environmental Health, v. 30, n. 3, p. 367-377, 2017.

GUPTA, S. Ergonomic applications to dental practice. Indian journal of Dentistry Researsch, v. 22, n. 6, p. 816-844. 2011. 
HAYES, M.; COCKRELL, D.; SMITH, D. A Systematic Review of Musculoskeletal Disorders Among Dental Professionals.International Journal of Dental Hygiene, v. 7, n. 3, p. 159-165, 2009.

KOTLIARENKO, A. et al. Distúrbios osteomusculares e fatores associados em cirurgiões dentistas do meio oeste do estado de Santa Catarina. Revista Odonto Ciência, v. 24, n. 2, 2009.

MARTINS, C. A.; FADEL, M. A. V.; RÉGIS-FILHO, G. I. Lesões por esforços repetitivos em cirurgiões- dentistas: um estudo de caso: síndrome do túnel carpal. Jornal Brasileiro de Clínica Odontológica Integrada, v. 7, n. 41, p. 363-367. 2003.

MAENO, M. et al. Lesões por esforços repetitivos (LER), distúrbios osteomusculares relacionados ao trabalho (DORT), dor relacionada ao trabalho: protocolos de atenção integral. Ministério da Saúde (Brasil), p. 1-49, 2012.

PEREIRA, A. S. et al. Estudo da prevalência de doenças ocupacionais em CirurgiõesDentistas de São José dos Campos. Odonto. v. 19, n. 37, p. 7-14. 2011.

PEREIRA, F. T. F.; LOPES, F. F.; OLIVEIRA, A. E. Distúrbios osteomusculares relacionados ao trabalho entre os cirurgiões-dentistas especialistas e generalistas. Revista Brasileira de Odontologia, v. 61, n. 3/4, p. 213-216. 2004.

PURIENE, A. et al. General health of dentists. Literature review. Stomatologija, v. 9, n. 1, p. 10-20, 2007.

PUTZ-ANDERSON, V.; BERNARD, B.; BURT, S. Musculoskeletal disorders and workplace factors.National Institute for Occupational Safety and Health (NIOSH), v. 104, 1997.

REGIS-FILHO, G. I.; MICHELS, G.; SELL, I. Lesões por esforços repetitivos/distúrbios osteomusculares relacionados ao trabalho de cirurgiões-dentistas: aspectos biomecânicos. Production, v. 19, n. 3, p. 569-580, 2009.

SANTOS FILHO, S. B.; BARRETO, S. M. Atividade ocupacional e prevalência de dor osteomuscular em cirurgiões-dentistas de Belo Horizonte, Minas Gerais, Brasil: contribuição ao debate sobre os distúrbios osteomusculares relacionados ao trabalho. Cadernos de saúde pública, v. 17, n. 1, p. 181-93, 2001.

SANTOS, A. F. et al. Benefícios da ginástica laboral na prevenção dos distúrbios osteomusculares relacionados ao trabalho. Arquivos de Ciências da Saúde da UNIPAR, v. 11, n. 2, 2007.

SANTOS, R. L. X. et al. Lesão por esforços repetitivos ( LER / DORT ) em cirurgiõesdentistas da Clínica Odontológica da Polícia Militar de Pernambuco. Odontologia Clínicocientífica, v. 12, n. 3, p. 177-187. 2013.

SILVA, M. S. P. et al. Health problems due to dental practice at the public service of Campina Grande / PB / Brazil.Brazilian dental science, v. 15, n. 1, p. 68-73, 2012.

40 Id on Line Rev. Mult. Psic. V.11, N. 38. 2017 - ISSN 1981-1179

Edição eletrônica em http://idonline.emnuvens.com.br/id 
SILVA, P. H. L.; JESUS, C. S. Sintomas osteomusculares em cirurgiões-dentistas da rede pública. Revista da AMRIGS, v. 57, n. 1, p. 44-48. 2013.

\section{Como citar este artigo (Formato ABNT):}

VIANA, Hugo V.; ROCHA, Marcelo P.da Lesões por Esforços Repetitivos e Distúrbios Osteomusculares em cirurgiões-dentistas. Id on Line Revista Multidisciplinar e de Psicologia, 2017, vol.11, n.38, p.28-41. ISSN: 19811179.

Recebido: 07.10.2017

Aceito: 09.10 .2017 